\title{
EDITORIAL
}

\section{Communicating with Authors}

In common with all scientific journals, communicating with authors is a major editorial activity at the British Journal of Nutrition. These communications are very important for the Journal for a number of reasons. One of the most important is that they provide the author with an impression of the general approach of the Editors and Editorial Staff to his or her paper. The quality and clarity of these communications are also critically important for the speed with which a paper can be handled because the communications must give the author a very clear idea of what we consider is required for the acceptance of a paper. As all authors will know, we customarily send an Editorial Report, prepared by the First Editor who is a specialist dealing with the scientific area, plus, where appropriate, a report from the Statistical Editor and a Referee's report. These are accompanied by a letter from the Chairman of the Editorial Board.

For many years we have used a series of 'standard letters' to communicate our decisions regarding the papers in these letters which accompany the reports. The letter and the reports are complementary and should always be read in conjunction with one another by the author or authors. The actual wording of the standard letters has been modified from time to time, primarily to make them more explicit.

Over the past few weeks, correspondence with one author has made it clear that the standard-letter approach can be misleading and it was suggested that it would be useful to discuss their use.

First, I think that it is important to say something about the nature of scientific papers. Although we follow the traditional format for a scientific paper and superficially the papers appear rather homogeneous, in fact each paper we receive is unique and, even with papers which the author sees as part of a series, has to be judged separately as an original, unique piece of scientific work. I think that the creative aspects of preparing a scientific paper of any kind are often not given sufficient attention, but that is possibly a topic for another occasion. This means that our views of each paper which have to be communicated to the author have to be specific for each paper. This is in essence what the Editorial Report and other reports provide. These reports can vary considerably in length and it is interesting to note that the length of the report bears no correlation with whether or not the paper is acceptable, or whether it requires major or minor revision for acceptance. This is not due to the idiosyncrasies of the different members of the Editorial Board but is a reflection of the uniqueness of each paper.

The spectrum of papers we receive ranges from the relatively few that are considered acceptable, as submitted, through to those that are judged as being inappropriate for the Journal and are rejected for this reason alone. Matching this spectrum of papers to a few standard letters is incredibly difficult, and in many cases it is not possible and a special letter has to be written; however, writing a special letter in many cases would merely involve reiteration of the points contained in the reports. In general we use up to four standard letters: accept as received; accept in principle, where the subject matter of the paper is appropriate but where there are a number of points which require attention (the overall criterion we use for this letter is that we expect the author to be able to deal with the points raised in one revision); the third category applies to where a paper is on an appropriate 
topic but where we need clarification or extension of the material to be able make a decision about acceptance (in some cases these papers may require a special letter to identify the really critical issues which the authors need to consider when revising the paper); the fourth standard letter deals with a revised paper which we believe needs further attention.

When I took over as Chairman of the Editorial Board I decided that the standard letter approach was inappropriate for a paper which we were rejecting. I know as an author that rejection is always a very unpleasant experience and I believe that the Journal has a responsibility to deal with rejection in a constructive and sensitive way. I do recognize that however carefully the letter is written it is still a rejection. As I have written earlier, there are many reasons for rejection, ranging from a judgement that a paper is inappropriate for the British Journal of Nutrition through to a paper which has been judged to be fatally flawed for some reason or other. In many cases the rejection applies to the form in which the paper is presented, which implies that the authors need to look very carefully at the paper as a whole and explore other ways of presenting the material. This process requires taking what may be a very radical view of the work, and for the majority of rejected papers our Editorial Reports contain suggestions as to how the authors may proceed.

Often when I see such papers I feel that if I or the Editor could sit down with the author for a day or so we could together produce an acceptable paper; if only the pressures of scientific life would allow this! I recognize that our Editorial Reports are a poor substitute for such a personal contact but they are the best we can do.

D. A. T. Southgate 